\title{
THE DEPTH OF AN IDEAL WITH A GIVEN HILBERT FUNCTION
}

\author{
SATOSHI MURAI AND TAKAYUKI HIBI
}

(Communicated by Bernd Ulrich)

\begin{abstract}
Let $A=K\left[x_{1}, \ldots, x_{n}\right]$ denote the polynomial ring in $n$ variables over a field $K$ with each $\operatorname{deg} x_{i}=1$. Let $I$ be a homogeneous ideal of $A$ with $I \neq A$ and $H_{A / I}$ the Hilbert function of the quotient algebra $A / I$. Given a numerical function $H: \mathbb{N} \rightarrow \mathbb{N}$ satisfying $H=H_{A / I}$ for some homogeneous ideal $I$ of $A$, we write $\mathcal{A}_{H}$ for the set of those integers $0 \leq r \leq n$ such that there exists a homogeneous ideal $I$ of $A$ with $H_{A / I}=H$ and with depth $A / I=r$. It will be proved that one has either $\mathcal{A}_{H}=\{0,1, \ldots, b\}$ for some $0 \leq b \leq n$ or $\left|\mathcal{A}_{H}\right|=1$.
\end{abstract}

\section{INTRODUCTION}

Let $A=K\left[x_{1}, \ldots, x_{n}\right]$ denote the polynomial ring in $n$ variables over a field $K$ with each $\operatorname{deg} x_{i}=1$. Let $I$ be a homogeneous ideal of $A$ with $I \neq A$ and $H_{R}$ the Hilbert function of the quotient algebra $R=A / I$. Thus $H_{R}(q), q=0,1,2, \ldots$, is the dimension of the subspace of $R$ spanned over $K$ by the homogeneous elements of $R$ of degree $q$. A classical result [3, Theorem 4.2.10] due to Macaulay guarantees that, given a numerical function $H: \mathbb{N} \rightarrow \mathbb{N}$, where $\mathbb{N}$ is the set of nonnegative integers, there exists a homogeneous ideal $I$ of $A$ with $I \neq A$ such that $H$ is the Hilbert function of the quotient algebra $R=A / I$ if and only if $H(0)=1, H(1) \leq n$ and $H(q+1) \leq H(q)^{\langle q\rangle}$ for $q=1,2, \ldots$, where $H(q)^{\langle q\rangle}$ is defined in [3, p. 161].

Given a numerical function $H: \mathbb{N} \rightarrow \mathbb{N}$ satisfying $H(0)=1, H(1) \leq n$ and $H(q+1) \leq H(q)^{\langle q\rangle}$ for $q=1,2, \ldots$, we write $\mathcal{A}_{H}$ for the set of those integers $0 \leq r \leq n$ such that there exists a homogeneous ideal $I$ of $A$ with $H_{A / I}=H$ and with depth $A / I=r$. We will show that, given a numerical function $H: \mathbb{N} \rightarrow \mathbb{N}$ satisfying $H(0)=1, H(1) \leq n$ and $H(q+1) \leq H(q)^{\langle q\rangle}$ for $q=1,2, \ldots$, one has (i) $\mathcal{A}_{H}=\{n-\delta\}$ if $H$ is of the form (1) of Proposition 1.5 and (ii) $\mathcal{A}_{H}=\{0,1, \ldots, b\}$ for some $b \geq 0$ if $H$ cannot be of the form (1). The statement (i) will be proved in Theorem 1.6, and the statement (ii) will be proved in Theorem 2.1. Also, we will introduce a way to determine the integer $b=\max \mathcal{A}_{H}$ from $H$ in Theorem 2.2.

Received by the editors August 9, 2006 and, in revised form December 5, 2006.

2000 Mathematics Subject Classification. Primary 13C15; Secondary 13D40.

Key words and phrases. Hilbert functions, depth, lexsegment ideals.

The first author is supported by JSPS Research Fellowships for Young Scientists. 


\section{UNIVERSAL LEXSEGMENT IDEALS}

Let $A=K\left[x_{1}, \ldots, x_{n}\right]$ denote the polynomial ring in $n$ variables over a field $K$ with each $\operatorname{deg} x_{i}=1$ and $A_{[m]}=K\left[x_{1}, \ldots, x_{n+m}\right]$, where $m$ is a positive integer. Work with the lexicographic order $<_{\operatorname{lex}}$ on $A$ induced by the ordering $x_{1}>x_{2}>$ $\cdots>x_{n}$ of the variables. Write, as usual, $G(I)$ for the (unique) minimal system of monomial generators of a monomial ideal $I$ of $A$. Recall that a monomial ideal $I$ of $A$ is lexsegment if, for a monomial $u$ of $A$ belonging to $I$ and for a monomial $v$ of $A$ with $\operatorname{deg} u=\operatorname{deg} v$ and with $v>_{\text {lex }} u$, one has $v \in I$. A lexsegment ideal $I$ of $A$ is called universal lexsegment ([1]) if, for any integer $m \geq 1$, the monomial ideal $I A_{[m]}$ of the polynomial ring $A_{[m]}$ is lexsegment. In other words, a universal lexsegment ideal of $A$ is a lexsegment ideal $I=\left(u_{1}, \ldots, u_{t}\right)$ of $A$ which remains being lexsegment if we regard $I=\left(u_{1}, \ldots, u_{t}\right)$ as an ideal of the polynomial ring $A_{[m]}$ for all $m \geq 1$.

Example 1.1. (a) The lexsegment ideal $\left(x_{1}^{2}, x_{1} x_{2}^{2}\right)$ of $K\left[x_{1}, x_{2}\right]$ is universal lexsegment. In fact, the ideal $\left(x_{1}^{2}, x_{1} x_{2}^{2}\right)$ of $K\left[x_{1}, \ldots, x_{m}\right]$ is lexsegment for all $m \geq 2$.

(b) The lexsegment ideal $\left(x_{1}^{3}, x_{1}^{2} x_{2}, x_{1} x_{2}^{2}\right)$ of $K\left[x_{1}, x_{2}\right]$ cannot be universal lexsegment. Indeed, since $x_{1} x_{2}^{2}<_{\text {lex }} x_{1}^{2} x_{3}$ in $K\left[x_{1}, x_{2}, x_{3}\right]$, the ideal $\left(x_{1}^{3}, x_{1}^{2} x_{2}, x_{1} x_{2}^{2}\right)$ of $K\left[x_{1}, x_{2}, x_{3}\right]$ is not lexsegment.

\section{Proposition 1.2.}

(a) Let $I$ be a lexsegment ideal of $A$ with $G(I)=\left\{u_{1}, \ldots, u_{\delta}\right\}$ where $\operatorname{deg} u_{1} \leq$ $\cdots \leq \operatorname{deg} u_{\delta}$ and where $u_{i+1}<_{\operatorname{lex}} u_{i}$ if $\operatorname{deg} u_{i}=\operatorname{deg} u_{i+1}$. Let $s_{1}=\operatorname{deg} u_{1}-1$ and $s_{i}=\operatorname{deg} u_{i}-\operatorname{deg} u_{i-1}$ for $i=2,3, \ldots, \delta$. Then, for $k \leq n$, one has

$$
u_{k}=x_{1}^{s_{1}} x_{2}^{s_{2}} \cdots x_{k}^{s_{k}+1} .
$$

(b) Given an integer $1 \leq \delta \leq n$ together with a sequence of integers $1 \leq e_{1} \leq$ $\cdots \leq e_{\delta}$, there is a lexsegment ideal $I$ of $A$ with $G(I)=\left\{u_{1}, \ldots, u_{\delta}\right\}$ such that $\operatorname{deg} u_{i}=e_{i}$ for $i=1, \ldots, \delta$.

Proof. (a) Since $u_{1}=x_{1}^{\operatorname{deg} u_{1}}$, one has $u_{1}=x_{1}^{s_{1}+1}$. Let $1<k \leq \min \{n, \delta\}$ and suppose that $u_{k-1}=x_{1}^{s_{1}} x_{2}^{s_{2}} \cdots x_{k-1}^{s_{k-1}+1}$. Since the ordering of $u_{1}, u_{2}, \ldots, u_{\delta}$ implies that the monomial ideal $\left(u_{1}, \ldots, u_{k-1}\right)$ is lexsegment, the smallest monomial with respect to $<_{\text {lex }}$ of degree $\operatorname{deg} u_{k}$ belonging to $\left(u_{1}, \ldots, u_{k-1}\right)$ is $u_{k-1} x_{n}^{s_{k}}$. Since $u_{k}$ is the largest monomial with respect to $<_{\operatorname{lex}}$ which satisfies $\operatorname{deg} u_{k}=$ $\operatorname{deg}\left(u_{k-1} x_{n}^{s_{k}}\right)$ and $u_{k}<_{\operatorname{lex}} u_{k-1} x_{n}^{s_{k}}$, we have $u_{k}=\left(u_{k-1} / x_{k-1}\right) x_{k}^{s_{k}+1}$. Thus $u_{k}=$ $x_{1}^{s_{1}} x_{2}^{s_{2}} \cdots x_{k-1}^{s_{k-1}} x_{k}^{s_{k}+1}$, as desired.

(b) This can be easily done by induction on $\delta$. Let $\delta \leq n$ and suppose that $J$ is a lexsegment ideal of $A$ with $G(J)=\left\{u_{1}, \ldots, u_{\delta-1}\right\}$ such that $\operatorname{deg} u_{i}=e_{i}$ for $i=1,2, \ldots, \delta-1$. Then by (a) we have $G(J) \subset K\left[x_{1}, \ldots, x_{\delta-1}\right]$. Hence $x_{\delta}^{e_{\delta}} \notin J$. Thus there exists a monomial of degree $e_{\delta}$ which does not belong to $J$. Let $u_{\delta}$ be the largest monomial of degree $e_{\delta}$ with respect to $<_{\operatorname{lex}}$ which does not belong to $J$. Then $\left(u_{1}, \ldots, u_{\delta-1}, u_{\delta}\right)$ is a lexsegment ideal of $A$.

Corollary 1.3. A lexsegment ideal $I$ of $A$ is universal lexsegment if and only if $|G(I)| \leq n$, where $|G(I)|$ is the number of monomials belonging to $G(I)$.

Proof. Let $G(I)=\left\{u_{1}, \ldots, u_{\delta}\right\}$, where $\operatorname{deg} u_{1} \leq \cdots \leq \operatorname{deg} u_{\delta}$. If $\delta \geq n+1$, then $I A_{[1]}$ is not a lexsegment ideal of $A_{[1]}$ since Proposition 1.2 (a) says that, for any lexsegment ideal $J$ of $A_{[1]}$ with $|G(J)| \geq n+1$, there exists a generator $v \in G(J)$ such that $x_{n+1}$ divides $v$. Thus $I$ is not a universal lexsegment if $\delta \geq n+1$. 
Assume that $\delta \leq n$. For any positive integer $m$, Proposition 1.2 (b) says that there exists the lexsegment ideal $J$ of $A_{[m]}$ such that $G(J)=\left\{v_{1}, \ldots, v_{\delta}\right\}$ satisfies $\operatorname{deg} v_{i}=\operatorname{deg} u_{i}$ for $i=1,2, \ldots, \delta$. Then Proposition 1.2 (a) says that $G(I)=G(J)$. Thus $I A_{[m]}$ is a lexsegment ideal of $A_{[m]}$ for all $m \geq 1$ if $\delta \leq n$.

For any monomial $u$ of $A$, let $m(u)$ be the biggest integer $1 \leq i \leq n$ for which $x_{i}$ divides $u$. A monomial ideal $I$ of $A$ is said to be stable if $u \in I$ implies $\left(x_{q} / x_{m(u)}\right) u \in$ $I$ for any $1 \leq q<m(u)$. Eliahou-Kervaire [5] says that, for a stable ideal $I$ of $A$, the projective dimension $\operatorname{proj} \operatorname{dim} A / I$ of the quotient algebra $A / I$ coincides with $\max \{m(u): u \in G(I)\}$. Since a lexsegment ideal is stable, it follows from Proposition 1.2 (a) together with the Auslander-Buchsbaum formula [3, Theorem 1.3.3] that

Corollary 1.4. Let $I$ be a lexsegment ideal of $A$ and $\operatorname{depth} A / I$ the depth of the quotient algebra $A / I$ of $A$. Then $\operatorname{depth} A / I=\max \{n-|G(I)|, 0\}$.

It is known that, given a numerical function $H: \mathbb{N} \rightarrow \mathbb{N}$ satisfying $H(0)=1$, $H(1) \leq n$ and $H(q+1) \leq H(q)^{\langle q\rangle}$ for $q=1,2, \ldots$, there exists a unique lexsegment ideal $I$ of $A$ with $H_{A / I}=H$. We say that a numerical function $H: \mathbb{N} \rightarrow \mathbb{N}$ satisfying $H(0)=1, H(1) \leq n$ and $H(q+1) \leq H(q)^{\langle q\rangle}$ for $q=1,2, \ldots$ is critical if the lexsegment ideal $I$ of $A$ with $H_{A / I}=H$ is universal lexsegment.

Proposition 1.5. A numerical function $H: \mathbb{N} \rightarrow \mathbb{N}$ satisfying $H(0)=1, H(1) \leq n$ and $H(q+1) \leq H(q)^{\langle q\rangle}$ for $q=1,2, \ldots$ is critical if and only if there is an integer $1 \leq \delta \leq n$ together with a sequence of integers $\left(e_{1}, \ldots, e_{\delta}\right)$ with $1 \leq e_{1} \leq \cdots \leq e_{\delta}$ such that

$$
H(q)=\left(\begin{array}{c}
n-1+q \\
n-1
\end{array}\right)-\sum_{i=1}^{\delta}\left(\begin{array}{c}
n-i+q-e_{i} \\
n-i
\end{array}\right)
$$

for $q=0,1, \ldots$ Moreover, $\delta$ is equal to the number of minimal monomial generators of the universal lexsegment ideal $I$ of $A$ with $H_{A / I}=H$.

Proof. First, to prove the "only if" part, let $I$ be a universal lexsegment ideal of $A$ with $G(I)=\left\{u_{1}, \ldots, u_{\delta}\right\}$, where $\delta \leq n$. Suppose that $\operatorname{deg} u_{1} \leq \cdots \leq \operatorname{deg} u_{\delta}$ and that $u_{i+1}<_{\operatorname{lex}} u_{i}$ if $\operatorname{deg} u_{i}=\operatorname{deg} u_{i+1}$. Proposition 1.2 (a) says that, for $1 \leq i<j \leq \delta$, the monomial $x_{i} u_{j}$ is divided by $u_{i}$ and no monomial belongs to both $u_{i} K\left[x_{i}, \ldots, x_{n}\right]$ and $u_{j} K\left[x_{j}, \ldots, x_{n}\right]$. Hence the direct sum decomposition $I=\bigoplus_{i=1}^{\delta} u_{i} K\left[x_{i}, \ldots, x_{n}\right]$ arises. Let $e_{i}=\operatorname{deg} u_{i}$ for $i=1,2, \ldots, \delta$. The fact that the number of monomials of degree $q$ belonging to $I$ is $\sum_{i=1}^{\delta}\left(\begin{array}{c}n-i+q-e_{i} \\ n-i\end{array}\right)$ yields the formula (1), as required.

Next we consider the "if" part. Let $H: \mathbb{N} \rightarrow \mathbb{N}$ be a numerical function of the form (1). Since $1 \leq e_{1} \leq \cdots \leq e_{\delta}$ and $\delta \leq n$, Proposition 1.2 (b) and Corollary 1.3 say that there exists a universal lexsegment ideal $I$ with $G(I)=\left\{u_{1}, \ldots, u_{\delta}\right\}$ such that $\operatorname{deg}\left(u_{i}\right)=e_{i}$ for all $i$. Then the computation of Hilbert functions in the proof of the "only if" part implies $H_{A / I}(q)=H(q)$ for all $q \in \mathbb{N}$.

A critical ideal of $A$ is a homogeneous ideal $I$ of $A$ with $I \neq A$ such that the Hilbert function $H_{R}$ of the quotient algebra $R=A / I$ is critical. In other words, a critical ideal of $A$ is a homogeneous ideal $I$ of $A$ such that the lexsegment ideal $I^{\text {lex }}$ is universal lexsegment, where $I^{\text {lex }}$ is the unique lexsegment ideal of $A$ such that $A / I$ and $A / I^{\text {lex }}$ have the same Hilbert function. Somewhat surprisingly, 
Theorem 1.6. Suppose that a homogeneous ideal I of $A$ is critical. Then

$$
\operatorname{depth} A / I=\operatorname{depth} A / I^{\text {lex }} .
$$

Proof. Let $\beta_{i j}$ (resp. $\beta_{i j}^{\prime}$ ) denote the graded Betti numbers of $I$ (resp. $I^{\text {lex }}$ ). Let $G\left(I^{\text {lex }}\right)=\left\{u_{1}, \ldots, u_{\delta}\right\}$ with $\delta \leq n$, where $\operatorname{deg} u_{1} \leq \cdots \leq \operatorname{deg} u_{\delta}$ and where $u_{i+1}<_{\text {lex }}$ $u_{i}$ if $\operatorname{deg} u_{i}=\operatorname{deg} u_{i+1}$. Let $e_{i}=\operatorname{deg} u_{i}$ for $i=1, \ldots, \delta$. Eliahou-Kervaire [5] together with Proposition 1.2 (a) guarantees that $\beta_{i, \delta-1+e_{\delta}}^{\prime}=0$ unless $i=\delta-1$ and $\beta_{\delta-1, \delta-1+e_{\delta}}^{\prime}=1$. Since $A / I$ and $A / I^{\text {lex }}$ have the same Hilbert function, it follows from [3, Lemma 4.1.13] that

$$
\sum_{i \geq 0}(-1)^{i} \beta_{i, \delta-1+e_{\delta}}=\sum_{i \geq 0}(-1)^{i} \beta_{i, \delta-1+e_{\delta}}^{\prime}
$$

Since $\beta_{i j} \leq \beta_{i j}^{\prime}$ for all $i$ and $j\left([2],[7]\right.$ and [8]), it follows that $\beta_{\delta-1, \delta-1+e_{\delta}}=1$. Thus in particular proj $\operatorname{dim} A / I \geq \delta$. Since $\operatorname{proj} \operatorname{dim} A / I^{\text {lex }}=\delta$ and $\operatorname{proj} \operatorname{dim} A / I \leq$ $\operatorname{proj} \operatorname{dim} A / I^{\text {lex }}$, it follows that $\operatorname{proj} \operatorname{dim} A / I=\operatorname{proj} \operatorname{dim} A / I^{\text {lex }}=\delta$. Thus we have $\operatorname{depth} A / I=\operatorname{dim} A / I^{\text {lex }}=n-\delta$, as desired.

Moreover, in the case of monomial ideals, the graded Betti numbers of a critical ideal are determined by its Hilbert function.

Corollary 1.7. Suppose that a monomial ideal I of $A$ is critical. Then $I$ and $I^{\text {lex }}$ have the same graded Betti numbers.

Proof. It follows from Taylor's resolution of monomial ideals (see [5, p. 18]) that

$$
\operatorname{proj} \operatorname{dim}(A / I) \leq|G(I)| \text {. }
$$

On the other hand, Corollary 1.4 and Theorem 1.6 say that

$$
\operatorname{proj} \operatorname{dim}(A / I)=\operatorname{proj} \operatorname{dim}\left(A / I^{\text {lex }}\right)=\left|G\left(I^{\text {lex }}\right)\right| \text {. }
$$

Since the number of elements in $G\left(I^{\text {lex }}\right)$ is always larger than that of $G(I)$, we have $|G(I)|=\left|G\left(I^{\text {lex }}\right)\right|$. This means $\sum_{j \geq 0} \beta_{0 j}(I)=\sum_{j \geq 0} \beta_{0 j}\left(I^{\text {lex }}\right)$. Then it follows from [4, Theorem 1.3] that $\beta_{i j}(I)=\beta_{i j}\left(I^{\text {lex }}\right)$ for all $i$ and $j$.

We are not sure that Corollary 1.7 holds for an arbitrary critical ideal.

Example 1.8. Let $I$ be the monomial ideal $\left(x_{1} x_{4}, x_{3} x_{4}\right)$ of $K\left[x_{1}, x_{2}, x_{3}, x_{4}\right]$. Since $I^{\text {lex }}=\left(x_{1}^{2}, x_{1} x_{2}\right)$ is universal lexsegment, it follows that depth $A / I=2$.

\section{Depth And Hilbert functions}

Let, as before, $A=K\left[x_{1}, \ldots, x_{n}\right]$ denote the polynomial ring in $n$ variables over a field $K$ with each $\operatorname{deg} x_{i}=1$. Given a numerical function $H: \mathbb{N} \rightarrow \mathbb{N}$ satisfying $H(0)=1, H(1) \leq n$ and $H(q+1) \leq H(q)^{\langle q\rangle}$ for $q=1,2, \ldots$, we write $\mathcal{A}_{H}$ for the set of those integers $0 \leq r \leq n$ such that there exists a homogeneous ideal $I$ of $A$ with $H_{A / I}=H$ and with $\operatorname{depth} A / I=r$. It follows from Corollary 1.4 together with Theorem 1.6 that if $H$ is critical, that is, $H$ is of the form (1), then $\mathcal{A}_{H}=\{n-\delta\}$.

Theorem 2.1. Suppose that a numerical function $H: \mathbb{N} \rightarrow \mathbb{N}$ satisfying $H(0)=1$, $H(1) \leq n$ and $H(q+1) \leq H(q)^{\langle q\rangle}$ for $q=1,2, \ldots$ is noncritical. Then $\mathcal{A}_{H}=$ $\{0,1,2, \ldots, b\}$, where $b$ is the largest integer for which $b \in \mathcal{A}_{H}$. 
Proof. We may assume that $K$ is infinite. Let $I$ be a homogeneous ideal of $A$ with $H_{A / I}=H$ and with $\operatorname{depth} A / I=b$. Let $0 \leq r \leq b$. Since $K$ is infinite and since $\operatorname{depth} A / I=b$, there exists a regular sequence $\left(\theta_{1}, \ldots, \theta_{r}\right)$ of $A / I$ with each $\operatorname{deg} \theta_{i}=$ 1. It then follows that there exists a homogeneous ideal $J$ of $B=K\left[x_{1}, \ldots, x_{n-r}\right]$ such that the ideal $J A$ of $A$ satisfies $H_{A /(J A)}=H$.

We now claim that the lexsegment ideal $J^{\text {lex }} \subset B$ of $J$ cannot be universal lexsegment. In fact, if $J^{\text {lex }}$ is universal lexsegment, then $J^{\text {lex }}$ remains being lexsegment in the polynomial ring $K\left[x_{1}, \ldots, x_{m}\right]$ for each $m \geq n-r$. In particular the ideal $J^{\text {lex }} A$ of $A$ is universal lexsegment. Since $H_{A /(J A)}=H_{A /\left(J^{\operatorname{lex}} A\right)}=H$, the numerical function $H$ is critical, a contradiction.

Since the lexsegment ideal $J^{\text {lex }}$ of $J$ cannot be universal lexsegment, it follows from Corollaries 1.3 and 1.4 that $\operatorname{depth} B / J^{\text {lex }}=0$. Thus $\operatorname{depth} A /\left(J^{\text {lex }} A\right)=r$. Hence $r \in \mathcal{A}_{H}$, as desired.

One may ask a way to compute the integer $b=\max \mathcal{A}_{H}$ from $H$. This integer $b$ can be determined as follows: Let $H: \mathbb{N} \rightarrow \mathbb{N}$ be a numerical function. The differential $\Delta^{1}(H)$ of $H$ is the numerical function defined by $\Delta^{1}(H)(0)=1$ and $\Delta^{1}(H)(q)=H(q)-H(q-1)$ for $q \geq 1$. We define the $p$-th differential $\Delta^{p}(H)=$ $\Delta^{1}\left(\Delta^{p-1}(H)\right)$ inductively.

Theorem 2.2. Let $H: \mathbb{N} \rightarrow \mathbb{N}$ be a numerical function satisfying $H(0)=1$, $H(1) \leq n$ and $H(q+1) \leq H(q)^{\langle q\rangle}$ for all $q \geq 1$. Then one has

(2) $\max A_{H}=\max \left\{p: \Delta^{p}(H)\right.$ satisfies $\Delta^{p}(H)(q+1) \leq \Delta^{p}(H)(q)^{\langle q\rangle}$ for $\left.q \geq 1\right\}$.

Proof. If $p$ is an integer which belongs to the right-hand side of (2), then there exists a homogeneous ideal $J$ of $B=K\left[x_{1}, \ldots, x_{n-p}\right]$ such that $H_{B / J}=\Delta^{p}(H)$. Recall that if $M$ is a graded $R$-module and $\vartheta_{1}, \ldots, \vartheta_{r}$ with each $\operatorname{deg} \vartheta_{i}=1$ is a regular sequence of $M$, then $H_{M /\left(\vartheta_{1}, \ldots, \vartheta_{r}\right) M}=\Delta^{p}\left(H_{M}\right)$. Set $M=A /(J A)$. Then, since $x_{n}, x_{n-1}, \ldots, x_{n-p+1}$ is a regular sequence of $A /(J A)$ and $M /\left(x_{n}, \ldots, x_{n-p+1}\right) M \cong$ $B / J$, we have $H_{A /(J A)}=H$ and $\operatorname{depth}(A /(J A)) \geq p$. This says that the left-hand side of (2) is greater than or equal to the right-hand side of (2).

On the other hand, if there exists a homogeneous ideal $I$ of $A$ such that $H=$ $H_{A / I}$ and $\operatorname{depth}(A / I)=p$, then, in the same way as Theorem 2.1, there exists a homogeneous ideal $J$ of $B=K\left[x_{1}, \ldots, x_{n-p}\right]$ such that $H_{A /(J A)}=H$ and $H_{B / J}=$ $\Delta^{p}(H)$. Thus the left-hand side of (2) is less than or equal to the right-hand side of (2).

Example 2.3. Let $I$ be the monomial ideal $\left(x_{1} x_{4}, x_{1} x_{5}, x_{2} x_{5}, x_{3} x_{5}, x_{2} x_{3} x_{4}\right)$ of $A=K\left[x_{1}, x_{2}, x_{3}, x_{4}, x_{5}\right]$. Then

$$
I^{\mathrm{lex}}=\left(x_{1}^{2}, x_{1} x_{2}, x_{1} x_{3}, x_{1} x_{4}, x_{1} x_{5}^{2}, x_{2}^{3}, x_{2}^{2} x_{3}, x_{2}^{2} x_{4}^{2}, x_{2}^{2} x_{4} x_{5}, x_{2}^{2} x_{5}^{3}, x_{2} x_{3}^{4}, x_{2} x_{3}^{3} x_{4}^{2}\right) .
$$

Thus depth $A / I^{\text {lex }}=0$ by Corollary 1.4. Since the Hilbert series $\sum_{q=0}^{\infty} H_{A / I}(q) \lambda^{q}$ of $A / I$ is $\left(1+2 \lambda-\lambda^{2}-\lambda^{3}\right) /(1-\lambda)^{3}$, it follows from [3, Corollary 4.1.10] that the Krull dimension of $A / I$ is 3 and $3 \notin \mathcal{A}_{H}$. Since depth $A / I=2$, one has $\mathcal{A}_{H}=\{0,1,2\}$.

\section{REFERENCES}

[1] E. Babson, I. Novik and R. Thomas, Reverse lexicographic and lexicographic shifting, J. Algebraic Combin. 23 (2006), 107-123. MR2223682 (2007a:13026)

[2] A. M. Bigatti, Upper bounds for the Betti numbers of a given Hilbert function, Comm. in Algebra 21 (1993), 2317-2334. MR1218500 (94c:13014) 
[3] W. Bruns and J. Herzog, "Cohen-Macaulay rings," Revised Edition, Cambridge University Press, 1998. MR1251956 (95h:13020)

[4] A. Conca, Koszul homology and extremal properties of Gin and Lex, Trans. Amer. Math. Soc. 356 (2004), no. 7, 2945-2961. MR2052603 (2005c:13012)

[5] S. Eliahou and M. Kervaire, Minimal resolutions of some monomial ideals, J. of Algebra 129 (1990), 1-25. MR1037391 (91b:13019)

[6] J. Herzog, Generic initial ideals and graded Betti numbers, in "Computational Commutative Algebra and Combinatorics" (T. Hibi, Ed.), Advanced Studies in Pure Math., Volume 33, 2002, pp. 75-120. MR1890097 (2003b:13021)

[7] H. A. Hulett, Maximum Betti numbers for a given Hilbert function, Comm. in Algebra 21 (1993), 2335-2350. MR1218501 (94c:13015)

[8] K. Pardue, Deformation classes of graded modules and maximal Betti numbers. Illinois J. Math. 40 (1995), 564-585. MR1415019 (97g:13029)

Department of Pure and Applied Mathematics, Graduate School of Information Science and Technology, Osaka University, Toyonaka, Osaka, 560-0043, Japan

E-mail address: s-murai@ist.osaka-u.ac.jp

Department of Pure and Applied Mathematics, Graduate School of Information Science and Technology, Osaka University, Toyonaka, Osaka, 560-0043, Japan

E-mail address: hibi@math.sci.osaka-u.ac.jp 